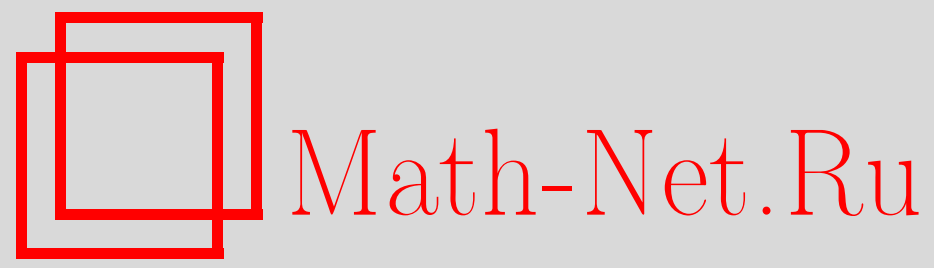

В. Л. Хацкевич, О средних значениях нечетких чисел и их систем, Нечеткие системы и мягкие вычисления, 2021, том 16, выпуск 1, 5-20

DOI: https://doi.org/10.26456/fssc75

Использование Общероссийского математического портала Math-Net.Ru подразумевает, что вы прочитали и согласны с пользовательским соглашением

http://www.mathnet.ru/rus/agreement

Параметры загрузки:

IP : 3.85 .183 .62

26 апреля 2023 г., 07:14:41 
УДК 519.766.2

О СРЕДНИХ ЗНАЧЕНИЯХ НЕЧЕТКИХ ЧИСЕЛ И ИХ СИСТЕМ

Хацкевич В.Л.

ВУНЦ ВВС Военно-воздушная академия имени профессора Н.Е. Жуковского и Ю.А. Гагарина, г. Воронеж

Поступила в редакцию 15.07.2020, после переработки 14.06.2021.

В настоящей работе рассмотрены экстремальные свойства средних значений нечетких чисел, а также их систем. Вводятся нелинейные средние и изучаются их свойства.

Ключевые слова: нечеткие числа, средние значения, экстремальные свойства.

Нечеткие системы и мягкие вычисления. 2021. Т. 16, № 1. С. 5-20. https://doi.org/10.26456/fssc75

\section{Введение}

Одним из разделов бурно развивающейся теории нечетких множеств является теория нечетких чисел.

Ниже, под нечетким множеством $A$, заданным на универсальном пространстве $X$, будем понимать совокупность упорядоченных пар $\left(\mu_{A}(x), x\right)$, где функция принадлежности $\mu_{A}(x): X \rightarrow[0,1]$, определяет степень принадлежности $\forall x \in X$ множеству $A$.

Мы будем использовать следующее определение нечеткого числа (ср. [1] гл. $2-4,[2] \S 1)$. Нечетким числом $A$ называется нечеткое множество, универсальным множеством которого является множество действительных чисел, и которое дополнительно удовлетворяет следующим условиям:

1. носитель нечеткого числа - замкнутое и ограниченное (компактное) множество действительных чисел: $\operatorname{Supp}(A) \subset R$;

2. функция принадлежности нечеткого числа $\mu_{A}(x)$ квазивогнута;

3. функция принадлежности нечеткого числа $\mu_{A}(x)$ нормальна;

4. функция принадлежности нечеткого числа $\mu_{A}(x)$ полунепрерывна сверху.

Мы опираемся на следующие свойства числовых средних.

Рассмотрим совокупность $n$ вещественных чисел $x_{1}, x_{2}, \ldots, x_{n}$. Как известно ([3] гл. $1, \S 5$, п. Б), их среднее $\bar{x}=\frac{1}{n} \sum_{k=0}^{n} x_{k}$ является решением следующей экстремальной задачи

$$
\sum_{i=0}^{n}\left(x_{i}-x\right)^{2} \rightarrow \min \quad(x \in R)
$$

(C) Хацкевич В.Л., 2021 
а медиана $M е$ является решением задачи

$$
\sum_{i=0}^{n}\left|x_{i}-x\right| \rightarrow \min (x \in R) .
$$

Такого рода экстремальные свойства и различные их обобщения иногда используются для самого определения средних значений объектов различного рода (см., напр., [3] гл. II, III, [4] гл. 5, §5).

В настоящей статье экстремальные свойства (в смысле минимизации некоторых расстояний) устанавливаются для средних нечетких чисел и их систем. При этом для нечетких чисел средние - это вещественные числа, а для систем нечетких чисел в качестве средних рассматриваются как вещественные, так и нечеткие числа. Кроме того, рассмотрены квазилинейные нечеткие средние. Приведены примеры.

Ниже в статье будут использованы различные определения и свойства общего характера из нечеткой арифметики. Весь цитируемый материал (кроме специальных вопросов) можно найти в [2]. Специальные вопросы по теме статьи цитируются по источникам.

\section{1. Средние нечетких чисел}

В литературе встречаются различные определения средних и медиан нечетких чисел (см., напр., [2] §1, [5] гл. 7).

Среднее значение нечеткого числа $A$, определяемого функцией принадлежности $\mu_{A}(x)$, задается с помощью метода центроидной дефаззификации следующим образом

$$
m_{1}(A)=\int_{-\infty}^{\infty} x \tilde{\mu}_{A}(x) d x
$$

где $\tilde{\mu}_{A}$ - нормированная функция принадлежности, определяемая формулой

$$
\tilde{\mu}_{A}=\frac{\mu_{A}(x)}{\int_{-\infty}^{\infty} \mu_{A}(x) d x} .
$$

Отметим, что интегрирование в (1) и (2) фактически осуществляется по $x \in \operatorname{supp}(A)$. Однако, для нас удобнее запись вида (1) и (2).

Экстремальное свойство среднего $m_{1}(A)$ аналогично стандартному свойству математического ожидания случайной величины с плотностью вероятности $\tilde{\mu}_{A}(x)$ (см., напр., [6] гл. $\left.5, \S 30\right)$. А именно, справедливо

Утверждение 1. Среднее $m_{1}(A)$, определяемое формулой (1), является решением, причем единственным, следующей экстремальной задачи

$$
\int_{-\infty}^{\infty}|x-y|^{2} \tilde{\mu}_{A}(x) d x \rightarrow \min \quad(y \in R),
$$

m.e.

$$
\int_{-\infty}^{\infty}\left|x-m_{1}(A)\right|^{2} \tilde{\mu}_{A}(x) d x \leq \int_{-\infty}^{\infty}(x-y)^{2} \tilde{\mu}_{A}(x) d x \quad(\forall y \in R) .
$$


Замечание 1. Пусть $\phi: R \rightarrow R$ заданная непрерывная строго монотонная числовая функция. Аналогично случайным величинам, для нечетких чисел можно определить и изучить класс нелинейньх средних вида $m_{\phi}(A)=\phi^{-1} \int_{-\infty}^{\infty} \phi(x) \tilde{\mu}(x) d x$.

Как показано в работе автора [7] нелинейное среднее $m_{\phi}(A)$ является решением экстремальной задачи $\int_{-\infty}^{\infty}(\phi(x)-y)^{2} \tilde{\mu}(x) d x \rightarrow \min (\forall y \in R)$.

Пример 1. Пусть треугольное нечеткое число $A$ характеризуется тройкой чисел $(a, b, c) a<b<c$, определяющей функцию принадлежности

$$
\mu_{A}(x)= \begin{cases}\frac{x-a}{b-a}, & \text { если } x \in[a, b] ; \\ \frac{x-c}{b-c}, & \text { если } x \in[b, c] ; \\ 0, & \text { в противном случае. }\end{cases}
$$

Нетрудно подсчитать, что нормированная функция принадлежности (2) имеет в этом случае вид $\tilde{\mu}(x)=\frac{2}{c-a} \mu(x)$. Тогда среднее значение треугольного числа, определяемое формулой $(1)$, равно $m_{1}(A)=\frac{1}{3}(a+b+c)$.

Медиану $M e A$ нечеткого числа $A$ по аналогии с медианой случайной величины можно определить как число, удовлетворяющее соотношению

$$
\int_{-\infty}^{M e A} \tilde{\mu}_{A}(x) d x=\frac{1}{2} .
$$

Утверждение 2. Медиана МеА нечеткого числа А, определяемая формулой (3), является решением экстремальной задачи

$$
\int_{-\infty}^{\infty}|x-y| \tilde{\mu}_{A}(x) d x \rightarrow \min \quad(y \in R) .
$$

Доказательство аналогично приводимому для случайных величин с плотностью распределения вероятностей $\tilde{\mu}_{A}(x)$ (см., напр., [6], гл. 5, 30).

Замечание 2. По аналогии с (3) можно ввести понятие квантиля $q_{\beta}$ нечеткого числа порядка $\beta$, определяемого для заданного $\beta \in(0,1)$ равенством $\int_{-\infty}^{q_{\beta}} \tilde{\mu}_{\beta}(x) d x=\beta$.

Согласно работе автора [8] квантиль минимизирует по $c \in(-\infty,+\infty)$ выражение

$$
\int_{-\infty}^{c}(c-x) \tilde{\mu}(x) d x+\frac{\beta}{1-\beta} \int_{c}^{+\infty}(x-c) \tilde{\mu}(x) d x .
$$

Пример 2. Рассмотрим треугольное нечеткое число $A$ с функцией принадлежности $\mu(x)$, представленной в примере 1 . В случае выполнения условия $\frac{1}{2}(a+c)>b$ медиана $M e A$ имеет вид $M e A=c-\sqrt{\frac{1}{2}(c-a)(c-b)}$. В противоположном случае, т.е., когда $\frac{1}{2}(a+c) \leq b$, медиана $M e A=a+\sqrt{\frac{1}{2}(c-a)(b-a)}$. 
С другой стороны, среднее значение нечеткого числа и его медиану можно определить посредством $\alpha$-уровневых множеств нечеткого числа.

Как известно, интервал $\alpha$-уровня нечеткого числа $A$ с функцией принадлежности $\mu_{A}(x)$ определяется следующим образом

$$
A_{\alpha}=\left\{x \mid \mu_{A}(x) \geq \alpha\right\} \quad(\alpha \in(0,1]) .
$$

Обратно: нечеткое число по своим $\alpha$-уровням однозначно восстанавливается посредством декомпозиции (см., напр.,[2] §1).

Согласно предположениям 1) - 4) введения все $\alpha$-уровни нечеткого числа - замкнутые и ограниченные интервалы вещественной оси. Обозначим левую (нижнюю) границу интервала $a^{-}(\alpha)$, а правую (верхнюю) $-a^{+}(\alpha)$, т.о. $A_{\alpha}=\left[a^{-}(\alpha), a^{+}(\alpha)\right]$. Иногда $a^{-}(\alpha)$ и $a^{+}(\alpha)$ называют, соответственно, левым и правым индексами нечеткого числа.

Как известно, среднее значение нечеткого числа $A$, используя интервальное представление, можно определить следующим способом

$$
m_{2}(A)=\frac{1}{2} \int_{0}^{1}\left(a^{-}(\alpha)+a^{+}(\alpha)\right) d \alpha
$$

Пример 3. Рассмотрим треугольное нечеткое число $A$, характеризуемое тройкой $(a, b, c)$. Как известно, в этом случае нижняя и, соответственно, верхняя граница $\alpha$-интервала имеет вид

$$
a^{-}(\alpha)=(b-a) \alpha+a, \quad a^{+}(\alpha)=-(c-b) \alpha+c .
$$

Тогда среднее (4) для нечеткого треугольного числа равно $m_{2}(A)=\frac{1}{4}(a+2 b+c)$.

Заметим, что согласно примерам 1 и 3 различным образом определяемые средние для заданного треугольного числа не совпадают.

На множестве нечетких чисел можно по-разному ввести определения расстояний между ними. Некоторые из них, определяемые посредством функций принадлежности (расстояние Евклида, расстояние Хэмминга), используются в п. 3. При интервальном подходе часто используют расстояние Хаусдорфа между множествами $\alpha$-уровня нечетких чисел (см., напр., [9] гл. 2, §2.5). Однако, хотелось бы рассмотреть такое определение расстояния, чтобы среднее (4) минимизировало некоторую среднеквадратическую ошибку (ср. [10] $\S 4.37,4.38)$. В качестве такого расстояния удобно рассмотреть следующее.

Пусть $A$ и $B$ два нечетких числа. Зададим расстояние $d(A, B)$ между ними формулой

$$
d(A, B)=\left(\int_{0}^{1}\left(a^{-}(\alpha)-b^{-}(\alpha)\right)^{2}+\left(a^{+}(\alpha)-b^{+}(\alpha)\right)^{2} d \alpha\right)^{1 / 2}
$$

Такое определение расстояния между нечеткими числами используется, например, в [11] (см. также [12]). Другие формулы для расстояний между нечеткими числами, выписываемые посредством функций принадлежностей, используются ниже.

Утверждение 3. Среднее значение (4) нечеткого числа А минимизирует величину

$$
d^{2}(A, y)=\delta(y)=\int_{0}^{1}\left(\left(a^{-}(\alpha)-y\right)^{2}+\left(a^{+}(\alpha)-y\right)^{2}\right) d \alpha \quad \forall y \in R
$$


причем оно является единственным решением этой экстремалъной задачи.

Доказательство следует из условия минимума для функции $\delta(y)$, т.к. $\delta^{\prime}(y)=4\left(y-m_{2}(A)\right)$, а вторая производная $\delta^{\prime \prime}(y)=4 \quad(\forall y \in R)$. Единственность влечет сильная выпуклость функции $\delta(y)$.

Несмотря на значительное число работ, посвященных средним нечетких чисел (см., напр., [13], [14]), экстремальные свойства средних чисел ранее не рассматривались.

Квазимедиану в случае интервального представления нечеткого числа $A$, используя принцип сохранения формальных свойств, естественно определить как решение следующей экстремальной задачи

$$
\Delta(y)=\int_{0}^{1}\left(\left|a^{-}(\alpha)-y\right|+\left|a^{+}(\alpha)-y\right|\right) d \alpha \rightarrow \min \quad \forall y \in R .
$$

Отметим, что в силу предположения о квазивогнутости функции принадлежности нечеткого числа, функции $a^{-}(\alpha)$ и $a^{+}(\alpha)$, характеризующие нижнюю и верхнюю границы интервала $\alpha$-уровня, являются, соответственно, монотонно неубывающей и монотонно невозрастающей по $\alpha$.

Напомним, что нечеткое число $A$ с функцией принадлежности $\mu_{A}(x)$ называют унимодальным (см. [2] $\S 1$ ), если условие $\mu_{A}(x)=1$ справедливо только для одного значения $x \in R$. Такое значение $x$ называют модой или модальным значением.

Утверждение 4. Пусть $A$ - унимодальное число со строго квазивогнутой функиией принадлежности. Тогда его квазимедиана, определяемая как решение задачи (6), совпадает с модой.

Доказательство. Пусть $\mu_{A}(x)$ - непрерывная квазивогнутая функция принадлежности нечеткого числа $A$, отрезок $[a, c]$ - носитель нечеткого числа $A$ и $b \in[a, c]$ - мода этого нечеткого числа, т.е. $\mu_{A}(b)=1$. Обозначим $\mu_{A}(x)$ при $x \in[a, b]$ через $\mu_{b^{-}}(x)$ и при $x \in[b, c]$ через $\mu_{b^{+}}(x)$. В силу строгой квазивогнутости $\mu_{A}(x)$ функция $\mu_{b^{-}}(x)$ строго монотонно возрастает, а функция $\mu_{b^{+}}(x)-$ строго монотонно убывает (каждая на своей области определения). Поэтому границы $\alpha$-интервала нечеткого числа $A$ можно задать с помощью обратных функций: $a^{-}(\alpha)=\mu_{b^{-}}^{-1}(\alpha)$ и $a^{+}(\alpha)=\mu_{b^{+}}^{-1}(\alpha)$.

Зафиксируем число $y$. Тогда, согласно (6), можем записать

$$
\begin{gathered}
\Delta(y)=\int_{0}^{\mu_{b^{-}}(y)}\left(y-a^{-}(\alpha)\right) d \alpha+\int_{\mu_{b^{-}}(y)}^{1}\left(a^{-}(\alpha)-y\right) d \alpha+ \\
+\int_{0}^{\mu_{b^{+}}(y)}\left(a^{+}(\alpha)-y\right) d \alpha+\int_{\mu_{b^{+}}(y)}^{1}\left(y-a^{+}(\alpha)\right) d \alpha .
\end{gathered}
$$

Дифференцируя это выражение по $y$, получим $\Delta_{y}^{\prime}=2\left(\mu_{b^{-}}(y)-\mu_{b^{+}}(y)\right)$.

Отметим, что по определению функция $\mu_{b^{-}}(y)$ монотонно убывает, а $\mu_{b^{+}}(y)$ монотонно возрастает. Поэтому функция $\Delta_{y}^{\prime}(y)$ - монотонно убывает. Тогда, если $y_{*}$ решение уравнения $\Delta_{y}^{\prime}\left(y_{*}\right)=0$, то в силу монотонности $\Delta_{y}^{\prime}(y)<\Delta_{y}^{\prime}\left(y_{*}\right)=0$ при $y<y_{*}$ и $\Delta_{y}^{\prime}(y)>0$ при $y>y_{*}$. Таким образом выполнено условие минимума в $y_{*}$. 
Пример 4. Для треугольного нечеткого числа $A$, характеризуемого тройкой $(a, b, c)$ (см. пример 1), в соответствии с утверждением 4 квазимедиана совпадает с модой и определяется равенством $M e A=b$.

\section{2. Средние и нечеткие средние систем нечетких чисел}

Возможны различные подходы к определению нечеткого среднего совокупности нечетких чисел.

Мы будем рассматривать нечеткие средние как решения некоторых экстремальных задач, по аналогии со средним арифметическим действительных чисел.

Пусть имеются вещественные числа $\beta_{i} \in R(i=1, \ldots, n)$ такие, что $\beta_{i} \geq 0$, $\sum_{i=1}^{n} \beta_{i}=1$.

Рассмотрим взвешенное нечеткое среднее нечетких чисел $\tilde{z}_{1}, \ldots, \tilde{z}_{n}$

$$
\tilde{z}_{\mathrm{cp}}=\sum_{i=1}^{n} \beta_{i} \tilde{z}_{i}
$$

Такого рода суммы встречаются, например, при нечетком портфельном инвестировании, когда $\tilde{z}_{i}$ - нечеткие доходности отдельных финансовых активов, $\beta_{i}$ - их доли в портфеле ценных бумаг.

Отметим, что функция принадлежности нечеткого числа (7) может быть построена по функциям принадлежности нечетких чисел $\tilde{z}_{i}$ на основе принципа обобщения Л. Заде (см., напр.,[2] §3). Поэтому среднее значение и медиана нечет-

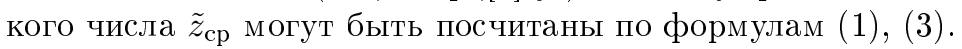

Остановимся более подробно на интервальном подходе. Рассмотрим среднее значение $(4) m_{2}\left(\tilde{z}_{\mathrm{cp}}\right)$ нечеткого числа $\tilde{z}_{\mathrm{cp}}$, определяемого формулой $(7)$.

Согласно утверждению 3 среднее $m\left(\tilde{z}_{\mathrm{cp}}\right)$ является решением следующей экстремальной задачи

$$
d\left(\tilde{z}_{\mathrm{cp}}, y\right) \rightarrow \min (\forall y \in R) .
$$

Здесь расстояние $d$ определяется формулой (5).

Кроме того, по определению интервального сложения, левый индекс $\tilde{z}_{\text {ср }}$ равен $z_{\mathrm{cp}}^{-}=\sum_{i=1}^{n} \beta_{i} z_{i}^{-}$, а правый индекс $-z_{\mathrm{cp}}^{+}=\sum_{i=1}^{n} \beta_{i} z_{i}^{+}$. Тогда согласно определению

$$
m_{2}\left(\tilde{z}_{\mathrm{cp}}\right)=\sum_{i=1}^{n} \beta_{i} m_{2}\left(\tilde{z}_{i}\right)
$$

В связи с этим имеет место

Теорема 1. Среднее значение $m_{2}\left(\tilde{z}_{c p}\right)$ нечеткого числа $\tilde{z}_{c p}$, определяемого формулой (7), является решением задачи

$$
\delta_{n}(y)=\sum_{i=1}^{n} \beta_{i} \int_{0}^{1}\left(\left(z_{i}^{-}(\alpha)-y\right)^{2}+\left(z_{i}^{+}(\alpha)-y\right)^{2}\right) d \alpha \rightarrow \min (\forall y \in R),
$$

причем единственным. Здесъ $z_{i}^{-}$и $z_{i}^{+}-$левый и правый индексы нечеткого числа $\tilde{z}_{i}$. 
Доказательство. Рассмотрим производную $\delta_{n}^{\prime}(y)$ :

$$
\begin{gathered}
\delta_{n}^{\prime}(y)=-2 \sum_{i=1}^{n} \beta_{i} \int_{0}^{1}\left(\left(z_{i}^{-}(\alpha)+\left(z_{i}^{+}(\alpha)-2 y\right) d \alpha=\right.\right. \\
=-2 \sum_{i=1}^{n} \beta_{i} \int_{0}^{1}\left(\left(z_{i}^{-}(\alpha)+\left(z_{i}^{+}(\alpha)\right) d \alpha+4 y .\right.\right.
\end{gathered}
$$

Поэтому условие $\delta_{n}^{\prime}\left(y_{*}\right)=0$ влечет равенство

$$
y_{*}=\sum_{i=1}^{n} \beta_{i} m_{2}\left(z_{i}\right) .
$$

Тогда согласно $(8) \delta_{n}^{\prime}\left(m_{2}\left(\tilde{z}_{\mathrm{cp}}\right)\right)=0$. При этом $\delta_{n}^{\prime \prime}(y)=4 \forall y \in R$. Так что утверждение теоремы есть следствие условия достаточности минимума для функции $\delta_{n}(y)$. Причем, единственность влечет сильная выпуклость функции $\delta_{n}(y)$.

Оказывается, что и само взвешенное нечеткое среднее (7) есть решение некоторой экстремальной задачи. А именно, справедлива

Теорема 2. Нечеткое среднее (7) является решением следующей задачи

$$
\sum_{i=1}^{n} \beta_{i} \int_{0}^{1}\left(\left(z_{i}^{-}(\alpha)-w^{-}(\alpha)\right)^{2}+\left(z_{i}^{+}(\alpha)-w^{+}(\alpha)\right)^{2}\right) d \alpha \rightarrow \min \quad(\forall \tilde{w} \in J),
$$

причем единственным. Здесь $J$ - совокупность всех нечетких чисел, обладающих свойствами 1)-4), представленных во введении.

Доказательство. Фиксируем $i$ и $\alpha \in(0,1]$. Аргумент $\alpha$ в нижеследующих формулах писать не будем, имея его в виду по умолчанию.

Рассмотрим выражение

$$
\begin{aligned}
\left(z_{i}^{-}-w^{-}\right)^{2}- & \left(z_{i}^{+}-w^{+}\right)^{2}=\left(z_{i}^{-}-z_{\mathrm{cp}}^{-}+z_{\mathrm{cp}}^{-}-w^{-}\right)^{2}+\left(z_{i}^{+}-z_{\mathrm{cp}}^{+}+z_{\mathrm{cp}}^{+}-w^{+}\right)^{2}= \\
= & \left(z_{i}^{-}-z_{\mathrm{cp}}^{-}\right)^{2}+\left(z_{\mathrm{cp}}^{-}-w^{-}\right)^{2}+2\left(z_{i}^{-}-z_{\mathrm{cp}}^{-}\right)\left(z_{\mathrm{cp}}^{-}-w^{-}\right)+ \\
& +\left(z_{i}^{+}-z_{\mathrm{cp}}^{+}\right)^{2}+\left(z_{\mathrm{cp}}^{+}-w^{+}\right)^{2}+2\left(z_{i}^{+}-z_{\mathrm{cp}}^{+}\right)\left(z_{\mathrm{cp}}^{+}-w^{+}\right) .
\end{aligned}
$$

Умножим обе части на $\beta_{i}$ и просуммируем по $i$ обе части полученного равенства. Тогда с учетом равенств

$$
\sum_{i=1}^{n} \beta_{i}\left(z_{i}^{-}-z_{\mathrm{cp}}^{-}\right)=\sum_{i=1}^{n} \beta_{i} z_{i}^{-}-z_{\mathrm{cp}}^{-}=0
$$

и

получим

$$
\sum_{i=1}^{n} \beta_{i}\left(z_{i}^{+}-z_{\mathrm{cp}}^{+}\right)=\sum_{i=1}^{n} \beta_{i}\left(z_{i}^{+}-z_{\mathrm{cp}}^{+}\right)=0
$$

$$
\sum_{i=1}^{n} \beta_{i}\left(\left(z_{\mathrm{cp}}^{-}-w^{-}\right)^{2}+\left(z_{\mathrm{cp}}^{+}-w^{+}\right)^{2}\right)=\sum_{i=1}^{n} \beta_{i}\left(\left(z_{i}^{-}-z_{\mathrm{cp}}\right)^{2}\right.
$$




$$
\left.\left.+\left(z_{i}^{+}-z_{\mathrm{cp}}\right)^{2}\right)+\left(z_{\mathrm{cp}}^{-}-w^{-}\right)^{2}+\left(z_{\mathrm{cp}}^{+}-w^{+}\right)^{2}\right) .
$$

Здесь мы учли, что $\sum_{i=1}^{n} \beta_{i}=1$.

Отсюда, после интегрирования по $\alpha$, следует высказанное утверждение.

Следствие 1. Нечеткое среднее $\hat{z}_{c p}=\frac{1}{n} \sum_{i=1}^{n} \tilde{z}_{i}$ является единственным решением экстремальной задачи

$$
\sum_{i=1}^{n} \int_{0}^{1}\left(\left(z_{i}^{-}(\alpha)-w^{-}(\alpha)\right)^{2}+\left(z_{i}^{+}(\alpha)-w^{+}(\alpha)\right)^{2}\right) d \alpha \rightarrow \min \quad(\forall \tilde{w} \in J) .
$$

Этот результат является обобщением классического характеристического экстремального свойства среднего арифметического. Автор к нему пришел в развитие своей работы [15].

Как известно, расстояние Евклида между нечеткими числами $A$ и $B$ с функциями принадлежности $\mu_{A}(x)$ и $\mu_{B}(x)$ определяется равенством

$$
d_{E}(A, B)=\left(\int_{-\infty}^{+\infty}\left(\mu_{A}(x)-\mu_{B}(x)\right)^{2} d x\right)^{1 / 2}
$$

Выпуклой комбинацией нечетких чисел $\tilde{z}_{i} i=1, \ldots, n$ с функциями принадлежности $\mu_{i}(x)$ называют нечеткое число $\tilde{z}$ с функцией принадлежности $\mu(x)=\sum_{i=1}^{n} \beta_{i} \mu_{i}(x)$. Здесь вещественные числа $\beta_{i} \in R(i=1, \ldots, n)$ такие, что $\beta_{i} \geq 0, \sum_{i=1}^{n} \beta_{i}=1$. Подчеркнем, что выпуклая комбинация $\mu(x)$, по существу, является взвешенным средним $\mu_{i}(x)$.

Обозначим через $\Phi_{\text {пр }}$ совокупность функций принадлежности, удовлетворяющих условиям 2) - 4) из определения нечеткого числа во введении.

Рассмотрим экстремальную задачу

$$
\sum_{i=1}^{n} \beta_{i} d_{E}^{2}\left(\tilde{z}_{i}, \tilde{z}_{\eta}\right) \rightarrow \min \left(\eta \in \Phi_{\text {пр }}\right)
$$

где $\tilde{z}_{\eta}$ - нечеткое число с функцией принадлежности $\eta(x)$.

Оказывается, что справедлива следующая

Теорема 3. Пусть заданы нечеткие числа $\tilde{z}_{i}(i=1, \ldots, n)$ с функииями принадлежности $\mu_{i}(x)$. Тогда их выпуклая комбинация является решением экстремальной задачи (11), причем единственным.

Доказательство. В соответствии с определением (10) экстремальная задача (11) может быть записана в виде

$$
\delta_{\eta}=\sum_{i=1}^{n} \beta_{i} \int_{-\infty}^{+\infty}\left(\mu_{i}(x)-\eta(x)\right)^{2} d x \rightarrow \min \quad\left(\eta \in \Phi_{\text {пр }}\right) .
$$


Пусть $\eta(x)$ - произвольная функция принадлежности из $\Phi_{\text {пр }}$, а $\mu(x)=\sum_{i=1}^{n} \beta_{i} \mu_{i}(x)$ - выпуклая комбинация функций принадлежности $\mu_{i}(x)$. Фиксируем $i$ и рассмотрим выражение

$$
\begin{gathered}
\left(\mu_{i}(x)-\eta(x)\right)^{2}=\left(\mu_{i}(x)-\mu(x)+\mu(x)-\eta(x)\right)^{2}= \\
=\left(\mu_{i}(x)-\mu(x)\right)^{2}+(\mu(x)-\eta(x))^{2}+2\left(\mu_{i}(x)-\mu(x)\right)(\mu(x)-\eta(x)) .
\end{gathered}
$$

Умножим обе части полученного равенства на $\beta_{i}$ и просуммируем их по $i$ от 1 до $n$. Тогда при каждом $x \in(-\infty,+\infty)$

$$
\begin{gathered}
\sum_{i=1}^{n} \beta_{i}\left(\mu_{i}(x)-\eta(x)\right)^{2}=\sum_{i=1}^{n} \beta_{i}\left(\mu_{i}(x)-\mu(x)\right)^{2} \\
+(\mu(x)-\eta(x))^{2}+2(\mu(x)-\eta(x)) \sum_{i=1}^{n} \beta_{i}\left(\mu_{i}(x)-\mu(x)\right) .
\end{gathered}
$$

При этом последнее слагаемое обращается в 0 по определению функции $\mu(x)$ и в силу равенства $\sum_{i=1}^{n} \beta_{i}=1$. Тогда

$$
\delta_{\eta}=\sum_{i=1}^{n} \beta_{i} \int_{-\infty}^{+\infty}\left(\mu_{i}(x)-\mu(x)\right)^{2} d x+\int_{-\infty}^{+\infty}(\mu(x)-\eta(x))^{2} d x
$$

Отсюда следует утверждение теоремы.

Следствие 2. Функция $\hat{\mu}(x)=\frac{1}{n} \sum_{i=1}^{n} \mu_{i}(x)$ минимизирует по $\eta$ величину $\sum_{i=1}^{n} \int_{-\infty}^{+\infty}\left(\mu_{i}(x)-\eta(x)\right)^{2} d x-$ сумму квадратов расстояний Евклида.

В силу общих соображений медианой совокупности нечетких чисел $\tilde{z}_{1}, \ldots, \tilde{z}_{n}$ можно было бы назвать медиану $M$ совокупности (обычных) чисел - медиан нечетких чисел $M e \tilde{z}_{1}, \ldots, M e \tilde{z}_{n}$. Как известно из классической статистики ([3] гл. $1, \S 5) M$ является решением следующей (числовой) экстремальной задачи

$$
\sum_{i=1}^{n}\left|M e \tilde{z}_{i}-y\right| \rightarrow \min \quad(\forall y \in R) .
$$

Одним из возможных подходов к определению нечеткой медианы является следующий. Ранжируем совокупность нечетких чисел $\tilde{z}_{1}, \ldots, \tilde{z}_{n}$, исходя из какоголибо признака сравнения (например, по средним значениям). Как обычно, срединное значение в полученном ряду назовем нечеткой квазимедианой.

С точки зрения экстремальных свойств (с учетом следствия 2) нечеткой квазимедианой совокупности нечетких чисел $\tilde{z}_{1}, \ldots, \tilde{z}_{n}$ с функциями принадлежности $\mu_{1}(x), \ldots, \mu_{n}(x)$ можно было бы считать нечеткое число, функция принадлежности $\mu(x)$ которого, минимизирует сумму расстояний Хэмминга

$$
\sum_{i=1}^{n} \int_{-\infty}^{\infty}\left|\mu_{i}(x)-\eta(x)\right| d x \longrightarrow \min \left(\forall \eta \in \Phi_{\text {пр }}\right) .
$$


Если использовать интервальный подход, то нечеткой квазимедианой совокупности нечетких чисел $\tilde{z}_{1}, \ldots, \tilde{z}_{n}$ (с учетом следствия 1 ) можно было бы считать нечеткое число, являющееся решением экстремальной задачи

$$
\sum_{i=1}^{n} \int_{0}^{1}\left(\left|z_{i}^{-}(\alpha)-w^{-}(\alpha)\right|+\left|z_{i}^{+}(\alpha)-w^{+}(\alpha)\right|\right) d \alpha \rightarrow \min \quad(\forall w \in J) .
$$

Другие определения нечетких медиан, не опирающиеся на экстремальные свойства, рассмотрены, например, в [5] гл. 7, [16]. По-видимому, выбор понятия медианы следует связывать с предметной областью исследования.

\section{3. Нелинейные нечеткие средние}

Перейдем к рассмотрению нелинейных нечетких средних систем нечетких чисел. Определим понятие функции от нечеткого числа, используя интервальный подход. Заметим, что этот подход к определению функции от нечеткого числа связан с принципом нечеткого обобщения Л. Заде (см., напр., [2] §3).

Пусть задана непрерывная монотонно возрастающая (монотонно убывающая) вещественная функция $\phi: D(R) \rightarrow R$ с областью определения $D(R)$

Лемма 1. Если $\tilde{z}$ - нечеткое число с левым и правым индексами $z^{-}(\alpha)$ и $z^{+}(\alpha)$ $u \phi(x)$ монотонно возрастающая функция, то $\phi\left(z^{-}(\alpha)\right)$ и $\phi\left(z^{+}(\alpha)\right)$ есть левый и правый индекс некоторого нечеткого числа $\tilde{w}_{\phi}\left(\right.$ так, что $w_{\phi}^{-}(\alpha)=\phi\left(z^{-}(\alpha)\right)$ u $\left.w_{\phi}^{+}(\alpha)=\phi\left(z^{+}(\alpha)\right)\right)$. Если $\phi(x)$ - монотонно убивающая функция, то $\phi\left(z^{+}(\alpha)\right)$ и $\phi\left(z^{-}(\alpha)\right)$ - левый и правый индексы, соответственно.

Это следует из того, что суперпозиция непрерывных функций непрерывна, суперпозиция монотонно возрастающих функций (либо монотонно убывающих) есть монотонно возрастающая (убывающая) функция, а суперпозиция монотонно убывающей и монотонно возрастающей (либо монотонно возрастающий и монотонно убывающей) есть монотонно убывающая функция.

Пусть заданы нечеткие числа $\tilde{z}_{1}, \ldots, \tilde{z}_{n}>0$, т.е. $z_{i}^{-}(\alpha)>0 \forall \alpha \in[0,1]$. А также действительные числа $\beta_{i} \in R\left(\beta_{i} \geq 0, \sum_{i=1}^{n} \beta_{i}=1\right)$. Рассмотрим нелинейные средние общего вида, для заданной монотонно возрастающей (убывающей) функции $\phi: R_{+} \rightarrow R:$

$$
\tilde{w}_{\phi}=\phi^{-1}\left(\sum_{i=1}^{n} \beta_{i} \phi\left(\tilde{z}_{i}\right)\right)
$$

Функцию $\phi$ в этом случае называют определяющей.

В случае определяющей функции $\phi_{p}(x)=x^{p}(p>1) \quad$ (или $\left.0<p<1\right)$ в (12) получаем среднюю степенную. Если $\phi_{G}(x)=\log _{a} x(a>1)$ (или $\left.0<a<1\right)$ - среднюю геометрическую, если $\phi_{H}(x)=\frac{1}{x}-$ среднюю гармоническую. Эти средние могут быть полезны в статистике нечетких данных, также как соответствующие им числовые нелинейные средние в классической статистике (см., напр., [3], [4]).

Согласно определению (12) из теоремы 2 следует 
Утверждение 5. Нечеткое число $\phi\left(\tilde{w}_{\phi}\right)$ минимизирует взвешенную сумму

$$
\sum_{i=1}^{n} \beta_{i} d^{2}\left(\tilde{z}_{i}, \tilde{z}\right) \quad(\forall \tilde{z} \in J)
$$

где $d$ - расстояние между нечеткими числами, определяемое формулой (5).

Коснемся вопроса о сравнении нечетких чисел. В литературе приняты различные показатели сравнения (ранжирования) нечетких чисел посредством функций принадлежностей (см., напр., [2] §8). Мы будем исходить из следующего.

Рассмотрим два нечетких числа $\tilde{z}$ и $\tilde{w}$, с соответствующими множествами $\alpha$ уровней $\left[z(\alpha)^{-}, z(\alpha)^{+}\right]$и $\left[w(\alpha)^{-}, w(\alpha)^{+}\right]$. Условие $\tilde{z}>\tilde{w}$ в классической традиции означает положительность нечеткого числа $\tilde{z}-\tilde{w}$. А оно в соответствие с интервальным определением вычитания обладает $\alpha$-уровневыми множествами вида $\left[z(\alpha)^{-}-w(\alpha)^{+}, z(\alpha)^{+}-w(\alpha)^{-}\right]$. В этом смысле неравенство $\tilde{z}-\tilde{w}>0$ эквивалентно условию $z(\alpha)^{-}-w(\alpha)^{+}>0(\forall \alpha \in(0,1]$.

Мы предлагаем рассмотреть следующее определение сравнения нечетких чисел, заданных в интервальной форме.

Будем писать $\tilde{z} \prec \tilde{w}$ для нечетких чисел $\tilde{z}$ и $\tilde{w}$, если одновременно

$$
z^{-}(\alpha) \leq w^{-}(\alpha), \quad z^{+}(\alpha) \leq w^{+}(\alpha) \quad(\forall \alpha \in(0,1]) .
$$

Ниже будут рассмотрены примеры, поясняющие смысл приведенных определений, для треугольных чисел.

Справедливо следующее соотношение типа (13) между нелинейными средними.

Теорема 4. Пусть $\tilde{z}_{1}, \ldots, \tilde{z}_{n}$ - совокупность нечетких чисел, $\phi(x)$ выпукло возрастающал функция, либо вогнуто убывающал функция. Тогда справедливо следующее соотношение

$$
\tilde{z}_{c p} \prec \tilde{w}_{\phi} .
$$

Здесь нечеткие средние $\tilde{z}_{c p}$ и $\tilde{w}_{\phi}$ определяются формулами (7) и (12), соответственно.

Если $\phi(x)$ выпуклая убывающая функиия, либо вогнутая возрастающая функиия, то

$$
\tilde{z}_{c p} \succ \tilde{w}_{\phi} .
$$

Действительно, рассмотрим первый случай. Надо показать, что

$$
z_{\mathrm{cp}}^{-}(\alpha) \leq w_{\phi}^{-}(\alpha), z_{\mathrm{cp}}^{+}(\alpha) \leq w_{\phi}^{+}(\alpha) \quad(\forall \alpha \in(0,1]) .
$$

Проведем рассуждения для левых индексов.

Заметим, что нечеткое число $\sum_{i=1}^{n} \beta_{i} \phi\left(\tilde{z}_{i}\right)$ в силу монотонного возрастания функции $\phi$ на основании леммы 1 и по правилу интервального сложения нечетких чисел и умножения их на положительные числа имеет левый индекс $\sum_{i=1}^{n} \beta_{i} \phi\left(z_{i}^{-}(\alpha)\right)$. Так как $\phi^{-1}$ также монотонно возрастающая функция, то нечеткое число $\phi^{-1}\left(\sum_{i=1}^{n} \beta_{i} \phi\left(\tilde{z}_{i}\right)\right)=\bar{w}_{\phi}$ имеет левый индекс $\phi^{-1}\left(\sum_{i=1}^{n} \beta_{i} \phi\left(z_{i}^{-}(\alpha)\right)\right)$.

Зафиксируем $\alpha \in(0,1]$. В силу свойства выпуклости вниз функции $\phi(x)$ функция $g(x)=\phi^{-1}(x)$ выпукла вверх. Тогда, как известно (см., напр., [17] гл. III), 
для любых чисел $y_{1}, \ldots, y_{n}$ и $\beta_{1}, \ldots, \beta_{n}$ таких что: $\beta_{i} \geq 0$ и $\sum_{i=1}^{n} \beta_{i}=1$ выполнено неравенство

$$
g\left(\sum_{i=1}^{n} \beta_{i} y_{i}\right) \geq \sum_{i=1}^{n} \beta_{i} g\left(y_{i}\right)
$$

Полагая здесь $y_{i}=\phi\left(z_{i}^{-}(\alpha)\right)$ и используя равенство $\phi^{-1}(\phi(y))=y$, получаем

$$
\phi^{-1}\left(\sum_{i=1}^{n} \beta_{i} \phi\left(z_{i}^{-}(\alpha)\right)\right) \geq \sum_{i=1}^{n} \beta_{i} z_{i}^{-}(\alpha) .
$$

То есть, соотношения (14) для левых индексов.

Аналогичные рассуждения справедливы для правых индексов.

В частности, теорема 4 справедлива при $\beta_{i}=\frac{1}{n}(i=1, \ldots, n)$, когда $\tilde{z}_{\text {ср }}$ есть средняя арифметическая.

Отметим случай, когда определяющая функция в (12) есть $\phi_{G}(x)=\log _{a}(x)$ $(a>1)$. Она вогнутая и монотонно возрастает. Тогда теорема 4 есть аналог знаменитого неравенства между средним арифметическим и средним геометрическим (см., напр., [17], гл. II, §2.5) для нечетких чисел.

Приведем примеры, раскрывающие смысл предлагаемых неравенств для треугольных нечетких чисел.

Пример 5. Для треугольных нечетких чисел $\tilde{z}=\left(a_{1}, b_{1}, c_{1}\right)$ и $\tilde{w}=\left(a_{2}, b_{2}, c_{2}\right)$ в соответствие с формулами для $\alpha$-уровневых множеств из примера 3 необходимым и достаточным условием выполнения соотношения $\tilde{z}>\tilde{w}$ является условие $a_{1}-c_{2}>0$.

Пример 6. В случае треугольных нечетких чисел $\tilde{z}=\left(a_{1}, b_{1}, c_{1}\right)$ и $\tilde{w}=\left(a_{2}, b_{2}, c_{2}\right)$ первое из соотношений (13) эквивалентно $a_{1} \geq a_{2}$, а второе - совокупности условий $c_{1} \geq c_{2}$ и $b_{1} \geq b_{2}$.

\section{Заключение}

В работе рассмотрены различные определения средних и медиан нечетких чисел. Проверены экстремальные свойства средних в смысле минимизации некоторых расстояний, когда сами средние можно определять как решения специально подобранных экстремальных задач. Рассмотрены средние и нечеткие средние систем нечетких чисел, а также нелинейные нечеткие средние. Установлен нечеткий аналог неравенства между средним арифметическим и средним геометрическим.

В литературе в качестве средних нечетких чисел иногда рассматриваются некоторые интервалы вещественной прямой (см., напр., [13]). В настоящей статье такой подход не анализируется. Приведенные в работе примеры основаны на треугольных нечетких числах. Они без труда распространяются на нечеткие числа (L-R)-типа и, в частности, трапецеидальные.

\section{Список литературы}

[1] Пегат А. Нечеткое моделирование и управление. М.: БИНОМ, 2015. 786 с.

[2] Борисов В.В., Федулов А.С., Зернов М.М. Основы нечеткой арифметики. Учебное пособие для вузов. М.: Горячая линия - Телеком, 2019. 98 с. 
[3] Джини К. Средние величины. М.: Статистика, 1970. 447 с.

[4] Орлов А.И. Прикладная статистика. М.: Издательство Экзамен, 2006. 672 с.

[5] Nguyen H.T., Wu B. Fundamentals of statistics with fuzzy data. Berlin: Springer, 2006. $204 \mathrm{p}$.

[6] Гнеденко Б.В. Курс теории вероятностей. М.: Книжный дом Либроком, 2011. $448 \mathrm{c.}$

[7] Khatskevich V.L. On some class of nonlinear mean random values // Journal of Physics: Conference Series. 2020. Vol. 1479. ID 012087. https://doi.org/10.1088/1742-6596/1479/1/012087

[8] Khatskevich V.L. On random properties of mean values of continuous random variables and relations between them // Journal of Mathematical Sciences. 2017. Vol. 1, № 2. Pp. 304-312.

[9] Язенин А.В. Основные понятия теории возможностей. Математический аппарат для принятия решений в условиях гибридной неопределенности. М.: Физматлит, 2016. 144 с.

[10] Hoffmann-Jorgensen J. Probability with a view toward statistics. New York: Chapman and Hall, 1998. 589 p.

[11] Bargiela A., Pedrycz W., Nakashima T. Multiple Regression with fuzzy data // Fuzzy Sets and Systems. 2007. Pp. 2169-2188.

[12] Вельдяксов В.Н., Шведов А.С. О методе наименьших квадратов при регрессии с нечеткими данными // Экономический журнал ВШЭ. 2014. Т. 18, № 2. C. $328-344$.

[13] Dubois D., Prade H. The mean value of fuzzy number // Fuzzy Sets and Systems. 1987. Pp. 279-300.

[14] Fuller R., Majlender P. On weighted possibilistic mean value and variance of fuzzy numbers // Fuzzy Sets and Systems. 2003. Pp. 363-374.

[15] Хацкевич В.Л. О некоторых экстремальных свойствах средних значений и математических ожиданиях случайных величин // Вестник Воронежского государственного технического университета. 2013. Т. 9, № 1-3. С. 39-44.

[16] Calvo T., Mesiar R. Generalized median // Fuzzy Sets and Systems. 2001. Vol. 124. Pp. 59-61.

[17] Харди Г., Пойа Д., Литтлвуд Д. Неравенства. М.: МЦНМО, 2008. 456 с.

\section{Образец цитирования}

Хацкевич В.Л. О средних значениях нечетких чисел и их систем // Нечеткие системы и мягкие вычисления. 2021. Т. 16, №1. С.5-20. https://doi.org/10.26456/fssc75 


\section{Сведения об авторах}

1. Хацкевич Владимир Львович сотрудник кафедры математики ВУНЦ ВВС ВВА имени профессора Н.Е. Жуковского и Ю.А. Гагарина.

Россия, 394052, г. Воронеж, ул. Маршала Неделина, 133. 


\title{
ON THE AVERAGE VALUES OF FUZZY NUMBERS AND THEIR SYSTEMS
}

\author{
Khatskevich Vladimir L'vovich
}

employee of the Department of Mathematics of Air Force Academy named after

N.E.Zhukovsky and Y.U. Gagarin

Russia, 394052, Voronezh, Marshala Nedelina str., 133.

Received 15.07.2020, revised 14.06.2021.

In this paper, we consider the extreme properties of the mean values of fuzzy numbers, as well as their systems. Nonlinear means are introduced and their properties are observed.

Keywords: fuzzy numbers, mean values, extreme properties.

\section{Citation}

Khatskevich V.L., "On the average values of fuzzy numbers and their systems", Nechetkie Sistemy i Myagkie Vychisleniya [Fuzzy Systems and Soft Computing], 16:1 (2021), 5-20(in Russian). https://doi.org/10.26456/fssc75

\section{References}

[1] Piegat A., Fuzzy Modeling and Control, Springer-Verlag, Berlin, Heidelberg, 2001, $725 \mathrm{pp}$.

[2] Borisov V.V., Fedulov A.S., Zernov M.M., Osnovy nechetkoj arifmetiki [Basics of fuzzy arithmetic], Tutorial, Goryachaya liniya - Telekom, Moscow, 2019 (in Russian), 98 pp.

[3] Dzhini K., Srednie velichiny [Average values], Statistika Publ., Moscow, 1970 (in Russian), 447 pp.

[4] Orlov A.I., Prikladnaya statistika, Ekzamen Publ., Moscow, 2006 (in Russian), $672 \mathrm{pp}$.

[5] Nguyen H.T., Wu B., Fundamentals of statistics with fuzzy data, Springer, Berlin, 2006, 204 pp.

[6] Gnedenko B.V., The Theory of Probability, AMS Chelsea Publishing, Providence, Rhode Island, 2005, $529 \mathrm{pp}$.

[7] Khatskevich V.L., "On some class of nonlinear mean random values", Journal of Physics: Conference Series, 1479 (2020), 012087, https://doi.org/10.1088/1742$6596 / 1479 / 1 / 012087$.

[8] Khatskevich V.L., "On random properties of mean values of continuous random variables and relations between them", Journal of Mathematical Sciences, 1:2 (2017), 304-312. 
[9] Yazenin A.V., Osnovnye ponyatiya teorii vozmozhnostej. Matematicheskij apparat dlya prinyatiya reshenij $v$ usloviyakh gibridnoj neopredelennosti [Basic concepts of the theory of possibilities. Mathematical decision-making apparatus in a hybrid uncertainty], Fizmatlit Publ., Moscow, 2016 (in Russian), 144 pp.

[10] Hoffmann-Jorgensen J., Probability with a view toward statistics, Chapman and Hall, New York, 1998, 589 pp.

[11] Bargiela A., Pedrycz W., Nakashima T., "Multiple Regression with fuzzy data", Fuzzy Sets and Systems, 2007, 2169-2188.

[12] Veldyaksov V.N., Shvedov A.S., "On the least squares method for regression with fuzzy data", Ekonomicheskij zhurnal VShE [HSE Economic Journal], 18:2 (2014), 328-344 (in Russian).

[13] Dubois D., Prade H., "The mean value of fuzzy number", Fuzzy Sets and Systems, 1987, 279-300.

[14] Fuller R., Majlender P., "On weighted possibilistic mean value and variance of fuzzy numbers", Fuzzy Sets and Systems, 2003, 363-374.

[15] Khatskevich V.L., "On some extreme properties of average values and mathematical expectations of random variables", Vestnik Voronezhskogo gosudarstvennogo tekhnicheskogo universiteta [Bulletin of the Voronezh State Technical University], 9:1-3 (2013), 39-44 (in Russian).

[16] Calvo T., Mesiar R., "Generalized median", Fuzzy Sets and Systems, 124 (2001), 59-61.

[17] Khardi G., Poja D., Littlvud D., Neravenstva [Inequalities], MTsNMO Publ., Moscow, 2008 (in Russian), 456 pp. 